Supplement of Clim. Past, 16, 1309-1323, 2020

https://doi.org/10.5194/cp-16-1309-2020-supplement

(C) Author(s) 2020. This work is distributed under

the Creative Commons Attribution 4.0 License.

(c) (1)

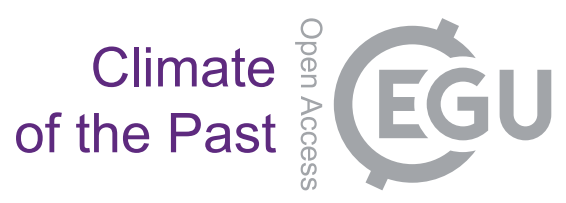

Supplement of

\title{
Assimilating monthly precipitation data in a paleoclimate data assimila- tion framework
}

Veronika Valler et al.

Correspondence to: Veronika Valler (veronika.valler@giub.unibe.ch)

The copyright of individual parts of the supplement might differ from the CC BY 4.0 License. 

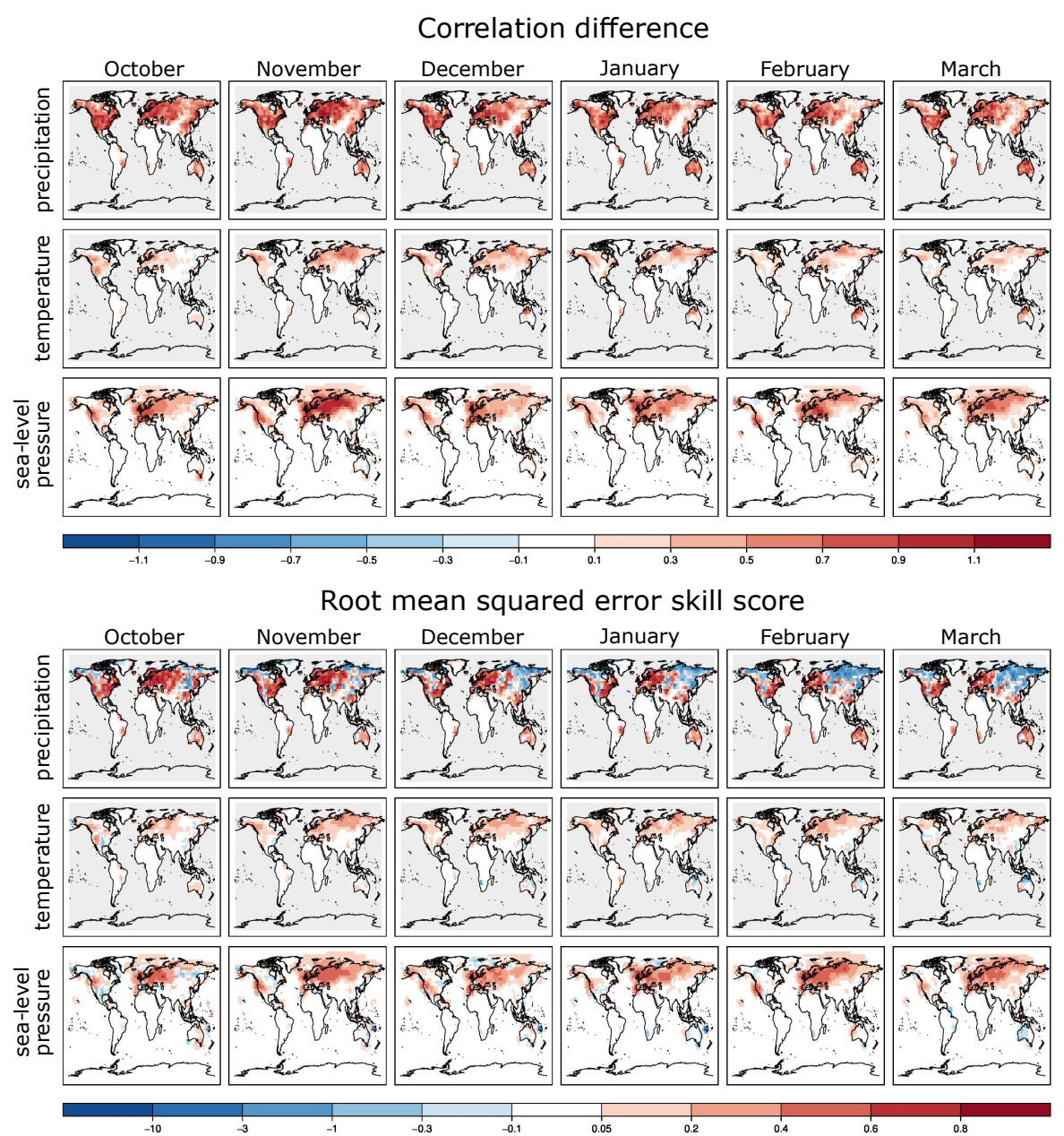

Figure S1. Monthly skill of the exp_R experiment from October to March. The correlation differences and RMSESS values of the precipitation, temperature and sea-level pressure reconstructions are shown. 

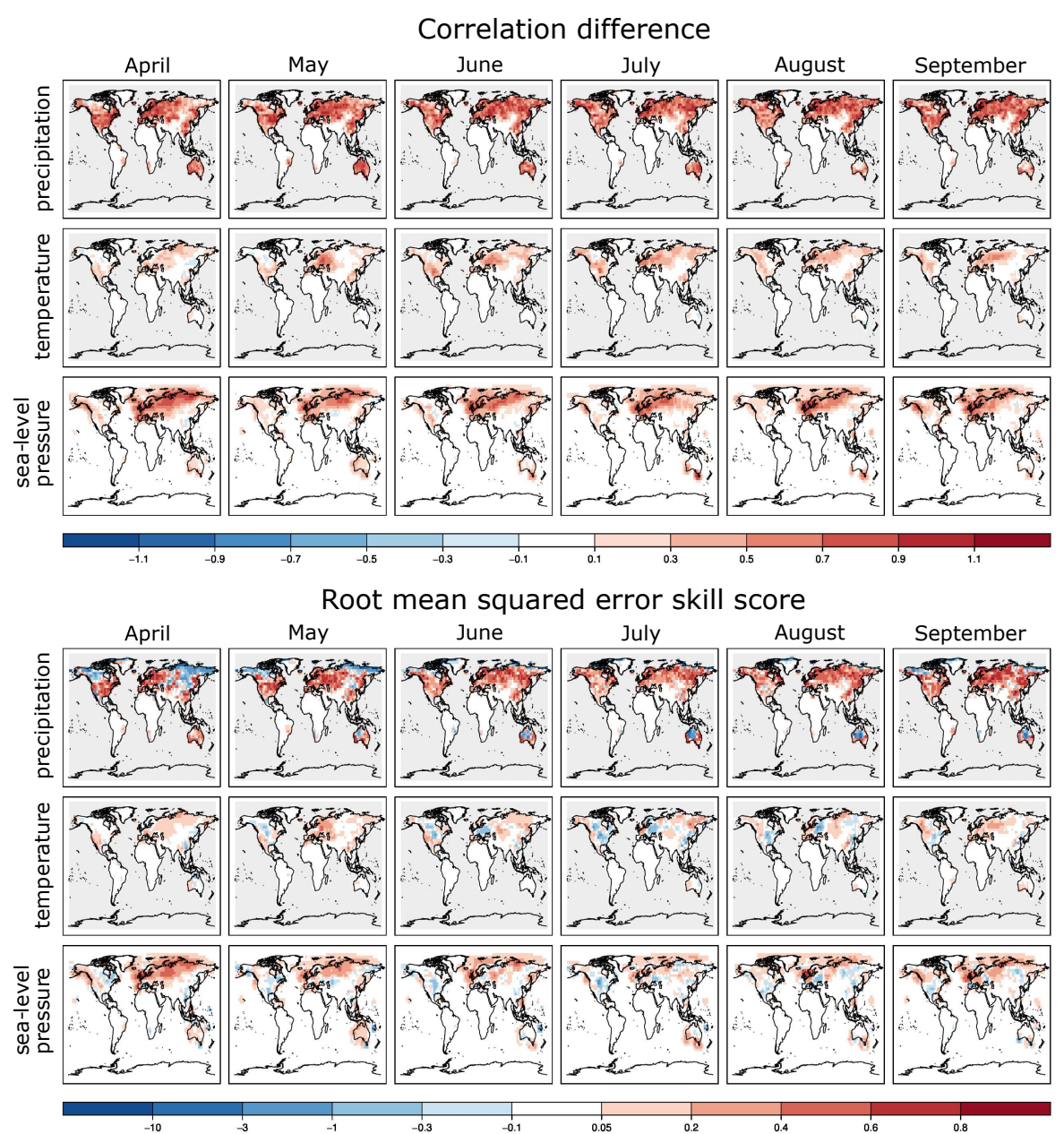

Figure S2. Monthly skill of the exp_R experiment from April to September. The correlation differences and RMSESS values of the precipitation, temperature and sea-level pressure reconstructions are shown. 


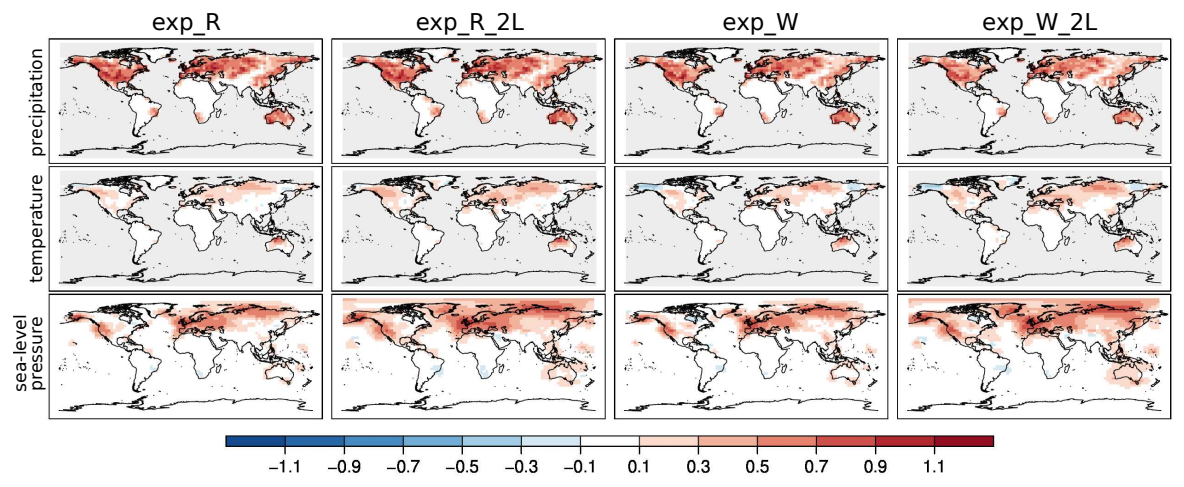

Figure S3. Winter season correlation differences of precipitation, of temperature, and of sea-level pressure between the analyses and the CCC400 model simulation ensemble means by assimilating only precipitation amounts, only precipitation amounts with doubled localization length scale parameter, only number of wet days, and only number of wet days with doubled localization length scale parameter.

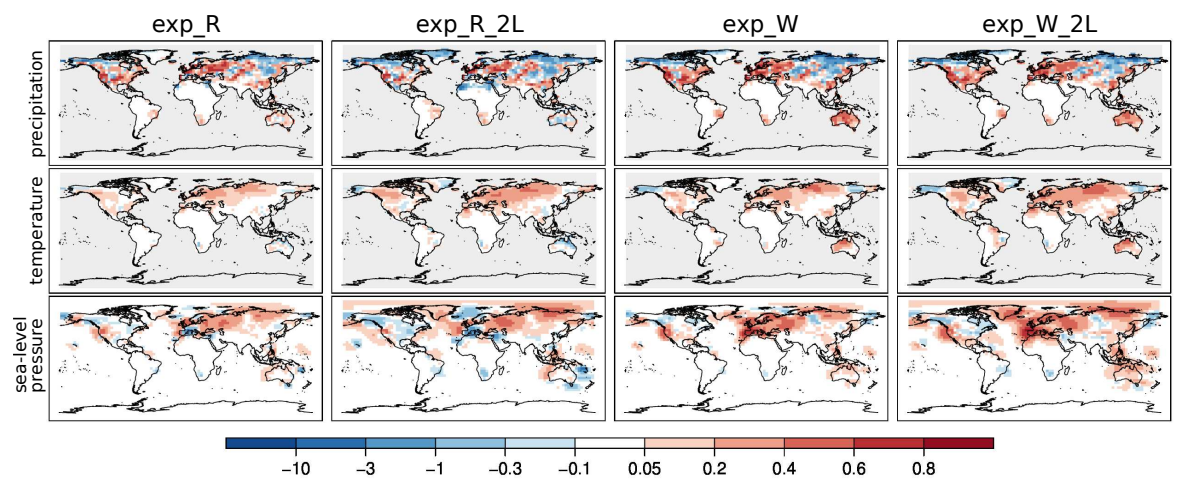

Figure S4. Winter season RMSESS values of precipitation, of temperature, and of sea-level pressure by assimilating only precipitation amounts, only precipitation amounts with doubled localization length scale parameter, only number of wet days, and only number of wet days with doubled localization length scale parameter. 


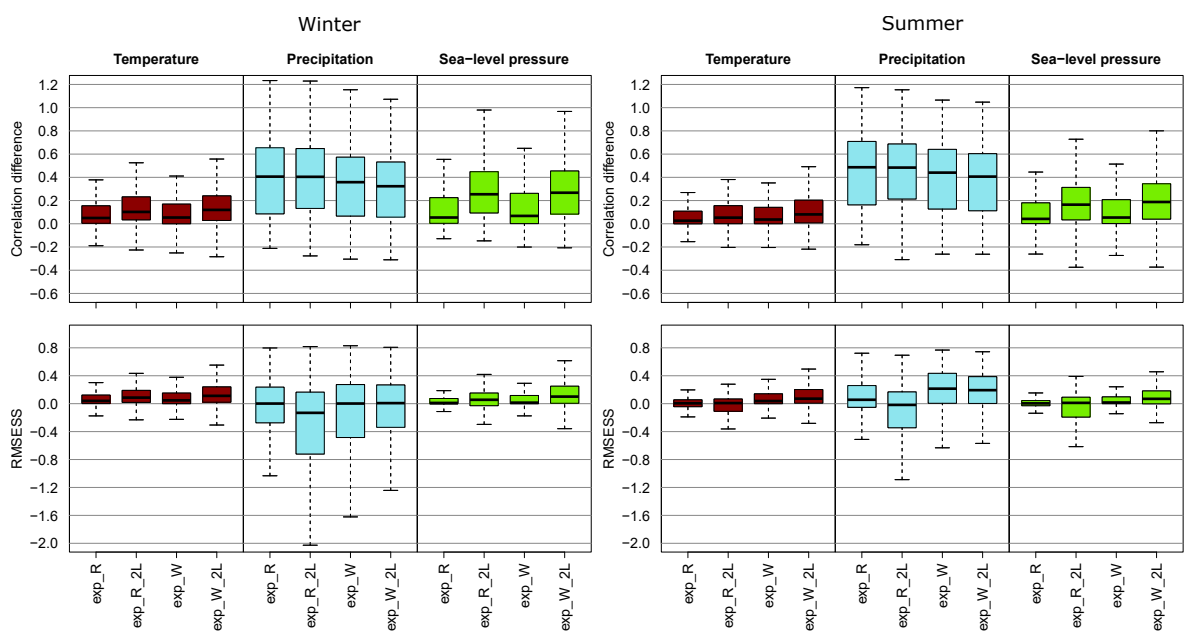

Figure S5. Distribution of skill metrics of exp_R, exp_R_2L, exp_W and exp_W_2L over the extratopical Northern Hemisphere in winter and summer. Correlation difference is calculated between the analysis ensemble mean and the refernce dataset subtracted the correlation between the model ensemble mean and the reference dataset. RMSESS is calculated according to Eq. (6), where $\boldsymbol{x}^{u}$ is the analysis ensemble mean of the experiments and $\boldsymbol{x}^{f}$ is the model ensemble mean. 


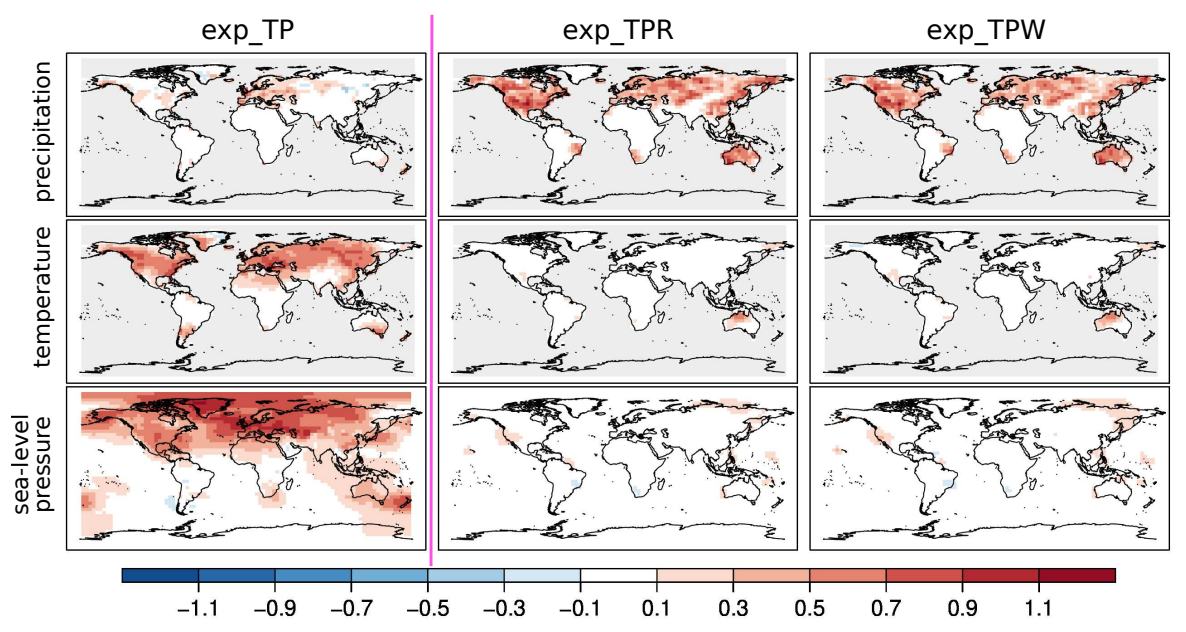

Figure S6. Winter season correlation differences between the analysis of exp_TP and the CCC400 model simulation ensemble means. In the middle column the correlation differences between the analyses ensemble mean of the exp_TPR and exp_TP are shown; while in the right column the correlation differences between the exp_TPR and exp_TP analyses ensemble mean are depicted.

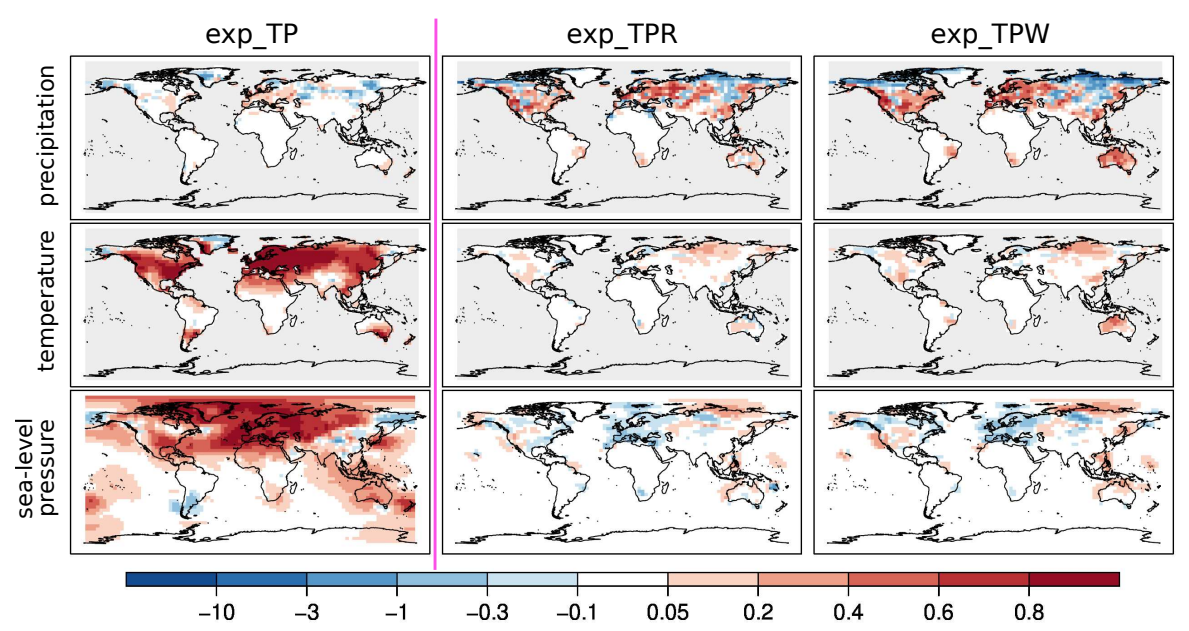

Figure S7. Winter season RMSESS values of exp_TP, exp_TPR and exp_TPW. $\boldsymbol{x}^{f}$ is the ensemble mean of the CCC400 model simulations when the RMSESS values calculated for the exp_TP experiment, while $\boldsymbol{x}^{f}$ was replaced with the analysis mean of the exp_TP for the other two experiments. 

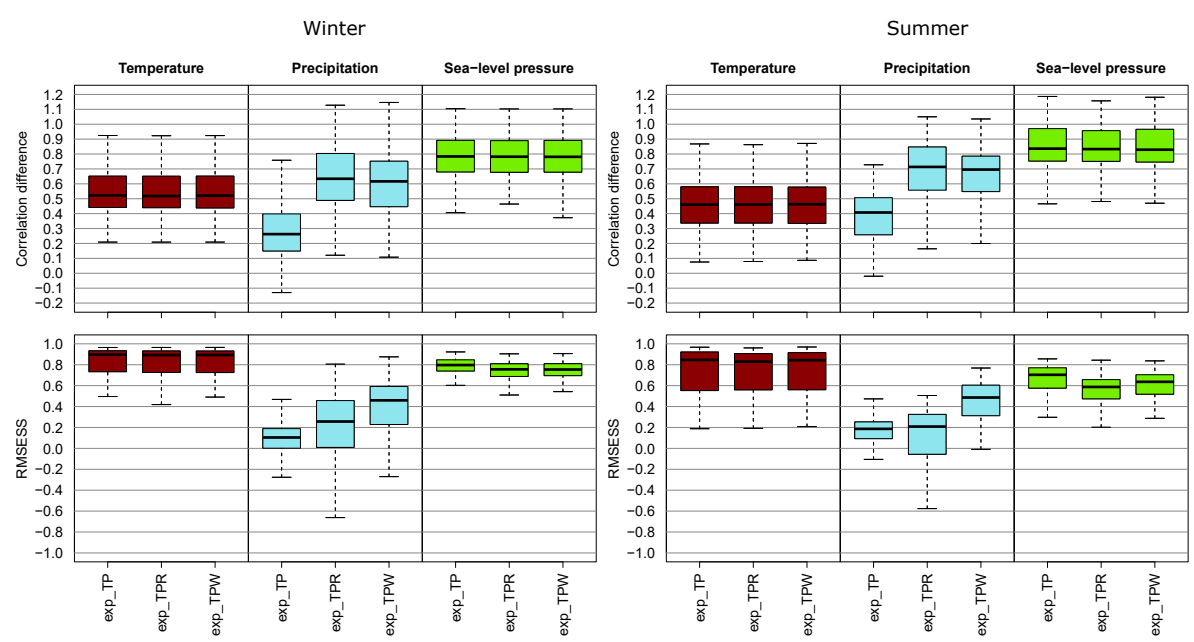

Figure S8. Distribution of skill metrics of exp_TP, exp_TPR and exp_TPW over Europe in winter and summer. Correlation difference is calculated between the analysis ensemble mean and the refeernce dataset subtracted the correlation between the model ensemble mean and the reference dataset. RMSESS is calculated according to Eq. (6), where $\boldsymbol{x}^{u}$ is the analysis ensemble mean of the experiments and $\boldsymbol{x}^{f}$ is the model ensemble mean. 


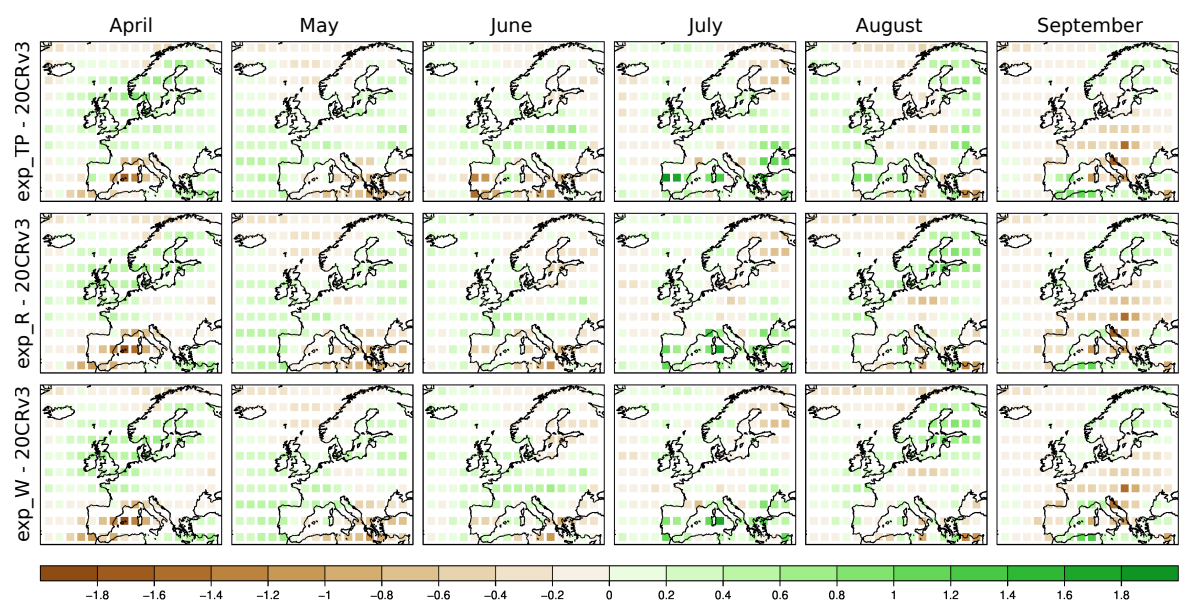

Figure S9. Differences of relative precipitation anomaly between the experiments and the 20CRv3 reanalysis over Europe in 1842. Monthly relative precipitation anomaly differences between April and September of exp_TP - 20CRv3 (top), exp_R - $20 \mathrm{CRv} 3$ (middle) and exp_W - 20CRv3 (bottom) are depicted. Note that the anomalies are calculated from 1807-1877 period for exp_TP, exp_R, and exp_W, and from 1836-1877 for 20CRv3. 


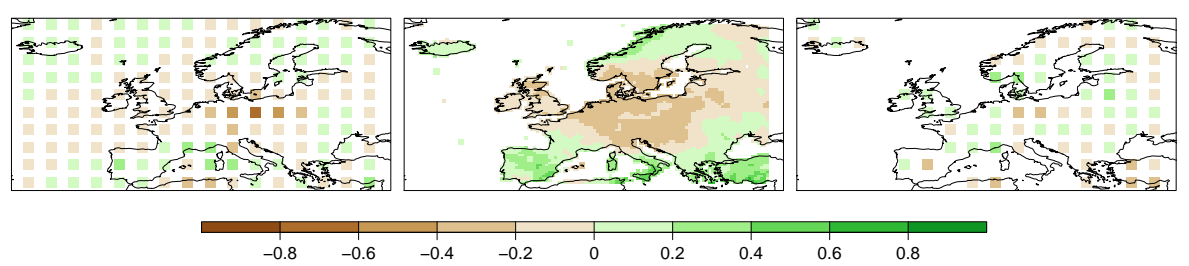

Figure S10. Relative precipitation anomaly over Europe in June-July-August in 1842, in exp_R (left), the reconstruction made by Pauling et al. (2006) (middle) and the differnce between the relative precipitation anomalies of exp_R and the reconstruction (right). 